\title{
Optimization of Cold Chain Path of Cooling Storage Multi - Temperature Fresh Produce under the Uncertain Demand and Time Window
}

\author{
CHEN KANG \\ Management School, Wuhan University of Science and Technology \\ Wuhan China \\ 818439111@qq.com
}

\begin{abstract}
In view of issues about the demand of various types of fresh agricultural products, the uncertainty of distribution time window and the difference of temperature layer in the existing cooling storage multi-temperature distribution process, cold chain path optimization model of multi - temperature fresh agricultural products with the demand and delivery time window of various kinds of fresh agricultural products subject to the known distribution is established. In the cold chain distribution, various costs incurred in the distribution are fully considered according to the characteristics of the cold chain, including transportation costs, carbon tax costs, incubator costs and time window penalty costs. Finally, a calculating example is used to verify the validity of the model. The results show that this model has good flexibility and applicability in the research of outlet demand and uncertainty of time window. Therefore, this model is worthy of promotion and reference.
\end{abstract}

Keywords-Uncertainty of demand and time window; Cooling storage multi-temperature allocation; Penalty cost of time window; Fresh agricultural produce

\section{INTRODUCTION}

With the rapid development of e-commerce in our country and the continuous improvement of people's living standards, the demand and types of fresh-cold chain products by urban residents are also increasing day by day. As an intermediary between business and customers, the operational efficiency and service levels of cold chain distribution are crucial. According to the statistics from the National Bureau of Statistics of China, the consumption of foodstuffs by residents nationwide increased by $7.1 \%$ and the per capita consumption expenditure by $6.8 \%$ in 2016 . Thus, people are more willing to pay for "eating". Online fresh cold chain food is a sudden emergence due to its convenient payment and rich variety. According to the statistics of China's TOT Cold Chain Commission, the market demand for the national cold chain logistics in 2016 reached 220 billion yuan, which was a year-on year growth of $22.3 \%$. The national cold storage is expected to rise by 3.05 million tons in 2016, bringing the total to 40.15 million tons, up by $8.2 \%$ year-on-year. What's more, the output of frozen foods has increased at a rate of $20 \%$, even at a high rate of $35 \%$ in the recent three years, much higher than the global average of $9 \%$ [2]. As the Boston Consulting Group (BCG) predicted in their recent" Report on China's Fresh Food Consumption
Trends in 2016", the size of the fresh food e-commerce industry will reach 600 billion yuan, and the penetration rate will increase to $15 \%-25 \%$ in 2020 [3]. Besides, insiders believe that China's cold chain logistics industry will maintain an average annual growth rate of $25 \%$ in the future, and by 2017 the market size will reach 470 billion yuan. The next 5-10 years will be the best strategic opportunity for the development of China's cold chain logistics. More and more listed companies are also beginning to deploy cold chain logistics. The cold chain will enter the 4.0 era and the market size will reach 3 trillion yuan. Therefore, China is gradually entering the era of rapid development of the chilled and frozen food market.

While cold chain logistics develops rapidly, the corresponding problems are exposed. Cold chain distribution is the core link in the cold chain logistics, during the process from the producer to the final consumer, more than $80 \%$ of the time spent on the cold chain products is in the distribution transportation. However, China's cold chain distribution equipment is outdated and the cold chain product loss is great, especially in the circulation. According to statistics, about 12 million tons of fruit and 130 million tons of vegetables, with a total value of 10 billion U.S. dollars, are wasted during distribution each year due to the problem of cold chain distribution in China. At present, about $80 \%$ the fruits, vegetables, meat and aquatic products are transported in traditional compartments (normal-temperature and insulation vehicles), while the cold chain circulation rate of meat and poultry in developed countries such as Europe and the US has reached $100 \%$ and that of vegetables and fruit also reached $95 \%$ or more. Shortage of cold chain logistics distribution has led to serious loss of cold chain products, which causes the seasonal fluctuations in prices of cold chain products, so that businesses have suffered huge losses.

In recent years there have been multi-temperature distribution logistics model, which integrates multi-temperature preservation technology, delivery logistics, information communication and other technology. While normaltemperature vehicles equipped with the cooling storage incubator are used to load normal-temperature fresh cold chain products, they can also load chilled and frozen fresh cold chain products, taking full advantage of the cargo capacity in the same direction, distributing the fresh cold chain products several distribution terminals need in one-time delivery. At 
present, studies have shown [4] that the newly-built cooling storage multi-temperature allocation system, instead of the traditional mechanical multi-temperature system, can not only save energy and reduce emissions and reduce the vehicle purchase costs, but also can preserve the quality and extend the distribution range, which is the new direction of the development of cold chain logistics. In addition, in real life, outlets' demand for various kinds of agricultural products and distribution time window is not fixed. Seasonal changes, shifts in consumer preferences, and differences in information held by upstream and downstream sites can all lead to changes in network demand and delivery time windows. When the outlet demand and delivery time window changes, if the service for outlets is in accordance with the original fixed distribution model service, it is difficult to meet the actual needs of the outlet and service satisfaction rate. In view of this, based on the literature [12-14], this paper further studies the agricultural produce cold chain distribution cooling storage multitemperature under uncertain outlet need and time window. Compared with the above literature, the contribution of this paper is: first, the research object uses the cooling storage multi-temperature allocation, involving a variety of types; Second, establish the cold chain path optimization model of of dynamic multi-temperature agricultural produce where various types of agricultural products demand and distribution time window are subject to the known distribution, and verify the validity of the model with the improved saving algorithm.

\section{MODEL ESTABLISHMENT}

\section{A. Problem Description}

Suppose a distribution center uses the cooling storage multi-temperature distribution model to distribute $s$ types of agricultural products of different types of stratum to multiple outlets at the same time. The locations of outlets are known. The demand and distribution time windows of various types of agricultural products are subject to a known distribution and the demand of various types of agricultural products, the requirements between outlets and the requirements of delivery time window by outlets are independent from one another. Under the constraints of satisfying demand of various types of agricultural products and satisfaction rate of delivery time window, the path optimization is carried out according to the known distribution they obey.

\section{B. Model Assumption}

(1) A distribution center, the total volume of which can meet the needs of all retailers;

(2) All refrigerated trucks depart from and finally get back to the distribution center;

(3) The number of retailers, demand, geographical location and time window are known, and any two retailers can communicate with each other;

(4) The distribution center adopts the strategy of vehicle distribution to the retailer's service, that is, each retailer only serves one car once, and all retailers can get the service.

(5) The flow of goods is one-way, that is, delivery only;
(6) All goods retailers need are provided by the distribution center; there is no swap between retailers;

(7) Delivery vehicle models are the same, that is, a single model delivery, and the delivery tasks of each line are in the charge of one car;

(8) The total customer demand on the shipping route is less than the capacity of one vehicle;

(9) The demand of each retailer is determined and relatively stable over a certain period of time.

\section{Related parameters}

$L=\{0,1,2 \ldots n\}$ represents the set of outlets, where 0 represents the distribution center; $\{1,2 \ldots m\}$ is the number of transport vehicles and represents the set of vehicles; $S$ is the type of agricultural products and $\{1,2, \ldots r\}$ represents the collection of agricultural products; $M, M_{s}$ are respectively the rated load of vehicles and the rated load of each type of agricultural products; $D_{i j}$ is the distance between the outlet $i$ and outlet $j ; S_{i j}$ is the path saving value, the path value a vehicle saves from the outlet $i$ through the outlet $j$; $S_{i j}=2\left(D_{0 i}+D_{0 j}\right)-\left(D_{0 i}+D_{i j}+D_{j 0}\right)=D_{0 i}+D_{0 j}-D_{i j}$ $; P_{s}$ is the unit cost of each type of agricultural products; $Q_{j k s}$ is the amount of the $S$ kind of agricultural product the vehicle $k$ distributes to the outlet $j ; u_{j s}$ is the mean of the distribution function of the need of the $S$ kind of agricultural product in outlet $j$ obeys; $\sigma_{j s}$ is the standard deviation of the distribution function of the need of the $S$ kind of agricultural product in outlet $j$ obeys; $T_{j k}$ is the time window the vehicle $k$ distributes to the outlet $j ; \mu_{j}$ is the mean of the distribution function the outlet $j$ requires the distribution time window to obey; $\sigma_{j}$ is the standard deviation of the distribution function the outlet $j$ requires the distribution time window to obey; $q_{s}$ is the service level of the $s$ kind of agricultural product the outlet requires to distribute; $q_{t}$ is the service level of service level the outlet requires; $X_{i j k}$ is the variable of 0,1 , if the vehicle runs on the road from the outlet $i$ to the outlet $j$, take 1 , otherwise take $0 ; Y_{j k}$ is the variable of 0,1 , if the vehicle $k$ distributes for the outlet $j$, take 1 , otherwise take 0 . 


\section{THE ESTABLISHMENT OF OBJECTIVE FUNCTIONS}

\section{A. Transportation Costs}

Vehicle transportation costs consist of fixed costs and variable costs. Fixed costs are not considered. The variable costs include the fuel cost of traveling the vehicle, passing tolls and maintenance fees, etc., which are directly proportional to the mileage. If the transport cost of each type of agricultural product of unit quality and distance is $c_{s}$, the transportation cost will be:

$$
C_{1}=\sum_{i=0}^{n} \sum_{j=0}^{n} \sum_{k=1}^{m} \sum_{s=1}^{r} X_{i j k} c_{S} Q_{j k s} D_{i j}
$$

\section{B. Carbon Tax Costs}

Cold chain logistics, as a high-energy-consuming and high-carbon emission business in the logistics industry, contradicts the theme of low-carbon economy with highcarbon emissions. Therefore, while rapidly developing, coldchain logistics should consider reducing carbon emissions, adopting green logistics and adapting to low-carbon trends. By taxing emission of $\mathrm{CO}_{2}$, environmental protection can be achieved. If $F$ is the amount of carbon emission of each type of agricultural product the vehicle delivers in unit quality and distance and $P_{c}$ is the carbon price, the carbon tax costs will be:

$$
C_{2}=P_{c} F \sum_{i=0}^{n} \sum_{j=0}^{n} \sum_{k=1}^{m} \sum_{s=1}^{r} X_{i j k} Q_{j k s} D_{i j}
$$

\section{Incubator Costs}

The incubator cost mainly refers to the cost of incubators cold storage incubator procurement, maintenance, depreciation and use of cold equipment and other costs. If $c_{b}$ is the cost of each incubator and $N_{k s}$ is the number of cold storage incubators used to load the first type of agricultural product on the vehicle, the incubator costs will be:

$$
C_{3}=\sum_{k=1}^{m} \sum_{s=1}^{r} Y_{j k} c_{b} N_{k s}
$$

\section{Time Window Penalty Costs}

If the designated distribution time window of the outlet $j$ is $\left[t_{j a}, t_{j b}\right]$ and the acceptable distribution time window of the outlet $j$ is $\left[t_{j A}, t_{j B}\right]$.s shown in the diagram below: if the arriving time of the vehicle is before $t_{j A}$ or after $t_{j B}$, the penalty cost will be high; if the arriving time is within $\left[t_{j a}, t_{j b}\right]$, the penalty cost will be 0 ; if the arriving time is between $\left[t_{j A}, t_{j a}\right]$ and $\left[t_{j b}, t_{j B}\right]$, there will be certain penalty cost. The penalty coefficient in the unit time is respectively $\lambda_{1}$ and $\lambda_{2}$. Build the penalty cost function of a single network time window:
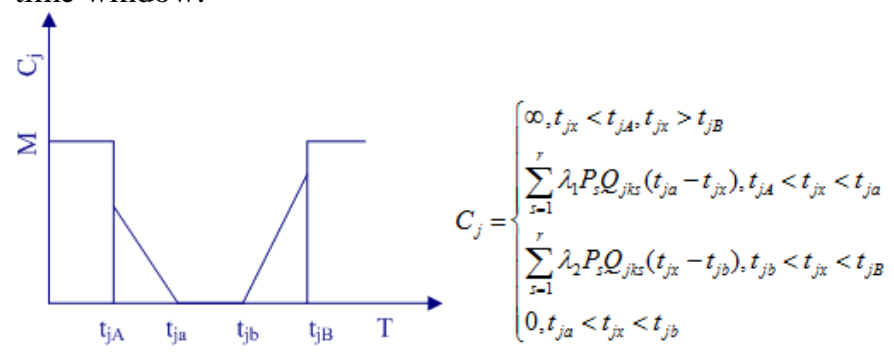

The time window penalty cost is:

$$
C_{4}=\sum_{j=0}^{n} C_{j}
$$

\section{E. Delivery Optimization Model}

In summary, the objective function is:

$$
\begin{aligned}
& \text { MinC }=C_{1}+C_{2}+C_{3}+C_{4}=\sum_{i=0}^{n} \sum_{j=0}^{n} \sum_{k=1}^{m} \sum_{s=1}^{r} X_{i j k} C_{s} D_{i j}+P_{c} F \sum_{i=0}^{n} \sum_{j=0}^{n} \sum_{k=1}^{m} \sum_{s=1}^{r} X_{i j k} Q_{j k s} D_{i j} \\
& +\sum_{k=1}^{m} \sum_{s=1}^{r} Y_{j k} c_{b} N_{k}^{s}+\sum_{j=0}^{n} C_{j}+\sum_{j=0}^{n} \sum_{s=1}^{r} C_{j}^{s} \\
& \text { st }: \sum_{j=0}^{n} \sum_{s=1}^{r} Y_{j k} Q_{j k s} \leq M, \forall k \\
& \sum_{j=0}^{n} Y_{j k} Q_{j k s} \leq M_{s}, \forall k, \forall s
\end{aligned}
$$

$$
t_{j A}<t_{j x}<t_{j B}
$$

$\sum_{j=0}^{n} \sum_{k=1}^{m} Y_{j k}=m, \forall k$

$\sum_{k=1}^{m} Y_{j k}=\left\{\begin{array}{l}1, j=1,2,3 \ldots m \\ m, j=0\end{array}\right.$

$\sum_{i=0}^{n} \sum_{j=0}^{n} X_{i j k}=2 n, \forall k$

$\sum_{j=0}^{n} \sum_{s=1}^{r} Q_{j k s} \geq \sum_{j=0}^{r} \sum_{s=1}^{r} u_{j s} \times Y_{j k}+Z_{s} \times \sqrt{\sum_{j=0}^{n} \sum_{s=1}^{r} \sigma_{j s}^{2} \times Y_{j k}}, \forall k$

$\sum_{j=0}^{n} T_{j} \geq \sum_{j=0}^{n} \mu_{j} \times Y_{j k}+Z_{t} \times \sqrt{\sum_{j=0}^{n} \sigma_{j}^{2} \times Y_{j k}}, \forall k$

Formula (1) is the optimization goal, which means the total cost is minimized; formula (2) is the vehicle load limit; formula (3) is the load limit of the first type of agricultural product; formula (4) is the time window limit; formula (5) is the available vehicles; formula (6) In order to ensure that each line starts and stops in the distribution center and each network only the only vehicle for its distribution; formula (7) To ensure that each outlet without omission distribution; 
formula (8) is The requirements of the outlets for the service rate of the first type of agricultural products shall be that the total load of the first type of agricultural products loaded on the vehicles must be greater than the minimum service level required of all kinds of agricultural products at the outlets to which it is responsible for distributing the services, formula (9) is the constraint on the time window service rate requirement of distribution of outlets, that is, the delivery time window of all the outlets in charge of vehicles must be greater than the minimum requirement of delivery time windows at the service level of all outlets that it is responsible for, Where is the distribution of the corresponding service level on the sub-site.

\section{APPLICATION EXAMPLES}

In order to verify the validity of the model, it is assumed that a distribution center distributes two different stratospheric agricultural products to 10 outlets. By the distribution center $\mathrm{q}$ $=24 \mathrm{t}$ vehicles to complete the transport mission, each cargo delivery volume of $12 \mathrm{t}$, the goods shelf life of $24 \mathrm{~h}$. In order to meet the demand of outlets, the distribution center required that all outlets meet the demand-satisfaction rate of each type of agricultural products and meet the delivery time window of $95 \%$, all of which are subject to independent normal distribution. 1,2 units of agricultural products quality, distance transportation costs are 1 yuan / tonne kilometers, the prices were 4000,3000 yuan / ton, unit time penalty coefficient $\lambda_{1}, \lambda_{2}$ are separately $0.2 \%, 3 \%$,each type of agricultural products unit penalty damage loss coefficient is 1 , outlets for each type of agricultural products acceptable loss rate upper and lower limits are $20 \%, 10 \%$, transport speed $30 \mathrm{Km} \mathrm{/} \mathrm{h,}$ loading and unloading time 20 minutes / customer, quality of each type of agricultural products, The distance of carbon emissions are 20 grams / tonne minute, carbon emissions price of 0.02 yuan / ton minute, the designated distribution time window of the outlet $j$ is $\left[t_{j A}, t_{j B}\right]$, and the acceptable distribution time window is $\left[t_{j A}-30, t_{j B}+30\right]$.The demand for various types of agricultural products at various outlets and the distance between the outlets and the distribution centers are shown in the charts 1 and 2.The distribution of demand and time window at 10 outlets.

TABLE I THE DISTRIBUTION OF DEMAND AND TIME WINDOW AT 10 OUTLETS

\begin{tabular}{|c|c|c|c|c|c|}
\hline Outlet & 1 & 2 & 3 & 4 & 5 \\
\hline $\operatorname{Agri-1-}\left(u, \sigma^{2}\right)$ & $(0.4,3)$ & $(0.3,4)$ & $(0.2,6)$ & $(0.5,1)$ & $(0.4,2)$ \\
\hline $\operatorname{Agri-2-}\left(u, \sigma^{2}\right)$ & $(0.6,4)$ & $(0.1,5)$ & $(0.4,3)$ & $(0.3,4)$ & $(0.5,1)$ \\
\hline Time-W- $\left(\mu, \sigma^{2}\right)$ & $(6: 15-6: 45,9)$ & $(7: 00-7: 30,8)$ & $(5: 30-6: 00,9)$ & $(7: 20-7: 50,16)$ & $(6: 10-6: 50,8)$ \\
\hline Outlet & 6 & 7 & 8 & 9 & 10 \\
\hline $\operatorname{Agri-1-}\left(u, \sigma^{2}\right)$ & $(0.2,4)$ & $(0.1,1)$ & $(0.5,1)$ & $(0.4,3)$ & $(0.3,2)$ \\
\hline $\operatorname{Agri-2}-\left(u, \sigma^{2}\right)$ & $(0.1,2)$ & $(0.4,4)$ & $(0.4,1)$ & $(0.5,2)$ & $(0.6,1)$ \\
\hline Time-W - $\left(\mu, \sigma^{2}\right)$ & $(6: 50-7: 20,16)$ & $(5: 50-6: 20,9)$ & $(7: 40-8: 10,8)$ & $(7: 20-7: 50,9)$ & $(7: 50-8: 20,9)$ \\
\hline
\end{tabular}

TABLE II DISTANCE OF 10 OUTLETS AND THE DISTRIBUTION CENTER

\begin{tabular}{llllll}
\hline Outlet & $\mathbf{0}$ & $\mathbf{1}$ & $\mathbf{2}$ & $\mathbf{3}$ & $\mathbf{4}$ \\
\hline $\mathbf{0}$ & 0 & & & & \\
$\mathbf{1}$ & 15 & 0 & & & \\
$\mathbf{2}$ & 10 & 12 & 0 & & \\
$\mathbf{3}$ & 8 & 9 & 4 & 0 & \\
$\mathbf{4}$ & 7 & 10 & 8 & 4 & 0 \\
$\mathbf{5}$ & 5 & 12 & 12 & 5 & 10 \\
$\mathbf{6}$ & 6 & 14 & 10 & 6 & 8 \\
$\mathbf{7}$ & 3 & 15 & 8 & 7 & 6 \\
$\mathbf{8}$ & 5 & 12 & 7 & 5 & 10 \\
$\mathbf{9}$ & 6 & 10 & 10 & 6 & 5 \\
$\mathbf{1 0}$ & 8 & 15 & 5 & 8 & 4 \\
\hline
\end{tabular}

For the model planning with uncertain demand and time window, when the distribution center satisfies the demand satisfaction rate and service time window of $95 \%$ for each kind of agricultural products at a given site, according to the

$\begin{array}{llllll}5 & 6 & 7 & 8 & 9 & 10\end{array}$

$5 \quad 0$

$4 \quad 7$

54

$6 \quad 6$

$\begin{array}{rrrrr}6 & 4 & 8 & 6 & 10\end{array}$

knowledge of probability theory, $X \sim N\left(u, \sigma^{2}\right)$; when $x=u+z_{a} \times \sigma$ by checking normal distribution table, 
we get $z_{a}=z_{0.05}=1.65$. Put the data into the model, seek solution with lingo programming, find the optimal total cost of yuan and reach the optimal delivery route as follows:

TABLE III ISTRIBUTION LINE ARRANGEMENT

\begin{tabular}{cccc}
\hline Line 1 & Line 2 & Line 3 & Line4 \\
\hline $0-3-1-2-0$ & $0-7-5-6-9-0$ & $0-4-10-0$ & $0-8-0$ \\
\hline
\end{tabular}

\section{CONCLUSIONS}

This paper studies the optimization of cold chain path of cooling-storage multi-temperature agricultural products under the uncertainty of demand and time window, and optimizes the distribution line by lingo programming. The research significance of this model lies in loosening the demand of outlets and the limitation of time window, increasing the applicability of route planning and making the distribution system more flexible. Therefore, this model is worth promoting and referencing.

\section{REFERENCES}

[1] Li Xuegong. Status and development trend of China's agricultural cold chain logistics [J]. Economic Logistics.2014,4:45-49.

[2] Lu Junjie, Sun Shuangshuang. Study on Vehicle Routing Optimization Based on Cold Chain Logistics Distribution of Fresh Agricultural Products [J]. Guangdong Agricultural Sciences,2013,40(9):178-181.

[3] Wang Shuyun, Sun Hong. Study on the Optimization of Multitemperature Allocating Paths for Cold Chain Products under Random Demand [J]. Industrial Engineering and Management,2016,21(2): 49-58.

[4] Lan Hui, He Qinfei. Optimization of Cold Chain Logistics Distribution Routing Considering Road Access [J]. Journal of Dalian Maritime University: Natural Science,2015,41(4):67-74.

[5] Xu Jiuqiang, Xing Peilong, KongPiushi, Liu Dapeng. Bidirectional Logistics Routing Optimization Based on Improved Ant Colony Algorithm [J]. Journal of Northeastern University (Natural Science), 2012,33(9):1240-1243.

[6] Zhang Yaming, Li Na. Study on the Optimization of Cold Chain Logistics VRP Model Based on Elite Singleton Genetic Algorithm [J]. Management science, 2016,46(4):87-96.

[7] CHO Y J,LI C C.Application of Multi-temperature Refrigerated container to Improve the Distribution of Cold Logistics[J]. Journal of the Eastern Asia Society for Transportation Studies. 2005,6:2794-2808.

[8] KUO J C,CHEN M C.Developing an advanced Multi-temperature Joint Distribution system for the Food Cold Chain [j]. Food Control,2010,21(4):559-566.

[9] HSU C I, LIU K P.A Model for Facilities Planning for Multitemperature Joint Distribution system [J]. Food Control, 201,22(12):1873-1882.

[10] Wang Shuyun, Zhao Min. Multi-temperature Allocating Dynamic Mechanism of Cooling Storage Cold Chain Logistics [J]. Journal of Highway and Transportation Research and Development,2012,29(2):144-148.

[11] Wang Shuyun, Sun Hong, Mu Jinjin, Jin Hui. Optimization and Benefit of Cold Chain Multi-temperature Allocation: A Comparative Study Based on Cold Storage and Mechanical [J]. Journal of Highway Communication Technology, 2016,33(3):146-153.

[12] SAZAR Z, MIRZAPOUR AL-E-HASHEM S M J,BABOLI A A Biobjective Stochastic Programming Model for a Centralized Green Supply Chain with Deteriorating Products 2014.

[13] Lei Wei. Research on Vehicle Routing Problem with Uncertain Demand [D]. Guangzhou: Sun Yat-sen University,2010.
[14] Wang Shuyun, SUN Hong. Planning of Cold Chain Goods Routing under Demand Change [J]. Highway Transportation Science and Technology,2014,31(8):144-150. J. Clerk Maxwell, A Treatise on Electricity and Magnetism, 3rd ed., vol. 2. Oxford: Clarendon, 1892, pp.68-73.

[15] I.S. Jacobs and C.P. Bean, "Fine particles, thin films and exchange anisotropy," in Magnetism, vol. III, G.T. Rado and H. Suhl, Eds. New York: Academic, 1963, pp. 271-350.

[16] K. Elissa, "Title of paper if known," unpublished.

[17] R. Nicole, "Title of paper with only first word capitalized," J. Name Stand. Abbrev., in press.

[18] Y. Yorozu, M. Hirano, K. Oka, and Y. Tagawa, "Electron spectroscopy studies on magneto-optical media and plastic substrate interface," IEEE Transl. J. Magn. Japan, vol. 2, pp. 740-741, August 1987 [Digests 9th Annual Conf. Magnetics Japan, p. 301, 1982].

[19] M. Young, The Technical Writer's Handbook. Mill Valley, CA: University Science, 1989. 